\title{
Out-of-Step Relays Testing Procedure
}

\author{
Antans Sauhats ${ }^{1}$, Andrejs Utans ${ }^{2}$, Gregory Pashnin ${ }^{3}$, Dmitrijs Antonovs ${ }^{4},{ }^{1-4}$ Riga Technical University
}

\begin{abstract}
The paper presents out-of-step (OOS) protection device testing methodology under close-to-real power system operation conditions. The power system stability modelling software is used as source of test signals. The accurate modelling of power system in conjunction with dynamical modelling features allows the correct choice of the most reliable OOS protection scheme. Methodology was applied for out-of-step relay "AGNA" testing and device settings verification.
\end{abstract}

Keywords - Power system stability, out-of-step relay, power system modelling software, relay testing, COMTRADE format.

\section{INTRODUCTION}

Power system is a subject to a wide range of small or large disturbances which occurs during steady state condition. The small disturbances such as load variation persist in power continually and power systems adjust to these changing conditions and continue to operate with nominal voltages and frequency. The large disturbances such as faults, loss of generation, excessive overload or lines switching can cause some part of power system to become unstable and the loss of the synchronism with remaining parts. When two areas of a power system lose synchronism, the areas must be separated from each other as quickly as possible to avoid equipment damage and possible power blackouts [1, 2]. The power system should be separated in predetermined locations to maintain a load-generation balance in each of the separated areas. The task of power system separation is accomplished with the out-of-step protection. The out-of-step (OOS) protection implementation principles are well - known $[2,5]$ and OOS protection devices are in use in the power system utility. While the out-of-step relaying philosophy is simple, it is often difficult to implement for a large power system because of its complexity and large variety of different operating conditions to be studied. A power system regimes simulation is a typical way to evaluate the behaviour of protection and automation devices under complex or nonstandard operating conditions. Power system electromagnetic processes simulation programs are widely used for protection relays testing purposes, but, when power system stability is under consideration then electromechanical processes should be simulated. The great advantage of EUROSTAG simulation program is that program covers full range of transient processes, mid and long-term power system stability could be studied and appropriate signals could be simulated. Despite the fact that EUROSTAG simulated signals are not intended for real devices testing, some efforts can be taken to overcome this shortage and implement EUROSTAG simulation results for real OOS relay testing. Paper presents the methods and tools which allows OOS protection to be evaluated using EUROSTAG modelling capabilities.

\section{OOS Protection Modelling Objectives and Deficiencies}

To get all the information needed for successful OOS protection scheme realization several studies are to be carried out [2]:

1. The selection of network locations for placement of OOS protection systems can best be obtained through transient stability studies covering many possible operating conditions.

2. The maximum rate of slip is typically estimated from angular change versus time plots from stability studies.

3. Determination of the optimal place of power system sectioning during an out-of-step condition is necessary. This will typically depend on the impedance between islands, the potential to attain a good load/generation balance, and the ability to establish stable operating condition of separated areas.

All these studies can be successfully accomplished simulating a variety of power system conditions which can affect the system stability [3]. The power system modelling software EUROSTAG enables the power system processes to be simulated with high precision and is especially effective when power system stability study is necessary.

Thus, the following information and tools are required:

1. to build an accurate power system model the precise technical information about all the elements the power system is composed off (transformers, generators, lines) is required;

2. a software that would simulate a variety of conditions which can affect power system stability;

3 . to verify the correct application of out-of-step protection scheme, an appropriate mathematical model of the OOS relay should be included in the stability simulation program;

4. a set of simulations is required in order to analyze the efficiency of the selected out-of-step protection scheme.

The accurate model of the OOS protection in conjunction with the power system dynamical modelling determines the choice of the most reliable OOS protection scheme and makes it possible to calculate appropriate OOS relay settings.

The precise technical data about high voltage apparatus are available from power system utility. The EUROSTAG software can be used as tool to build the complex power system models and to simulate the variety of scenarios for power system stability study. The OOS protection model can also be build using the EUROSTAG software if the OOS protection operation principle is known and a description of device operational algorithms is at hand. However, if the OOS 
protection model is built according to the protection operation manual, then it will be necessary to decide whether the model is absolutely equivalent to the real device or not. One may argue that the device and its model are not absolutely the same things. This is just because the mathematical/logical description of the protection operation algorithms provided by manufacturer and software/hardware realization of the same algorithms makes the difference. The differences in measurement/calculation precision of the model and its real counterpart usually are tolerable, but there exists the probability of rare software errors as well as other irregularities which exist only in the real device and can affect the device proper operation. Observing the statistical information about the cause of incorrect protection operation [4] (Fig. 1.) the following can be pointed out: more than $10 \%$ of all incorrect protection operations are due to incorrect device settings, about $20 \%$ are due to internal relay fault (hardware or software). Another $20 \%$ are marked as "reason unknown" - sometimes the software error detection is nearly impossible because it is hard to reconstruct the situation when this error becomes visible and affects the device operation. This kind of errors can be fixed only when the device operates with real signals under real or close to real operation condition.

Taking into account all the above said as well as the extreme importance of the out-of-step relaying, the following conclusion can be made: the concept of OOS device testing in real or close to real conditions may become imperative for successful and confident relaying.

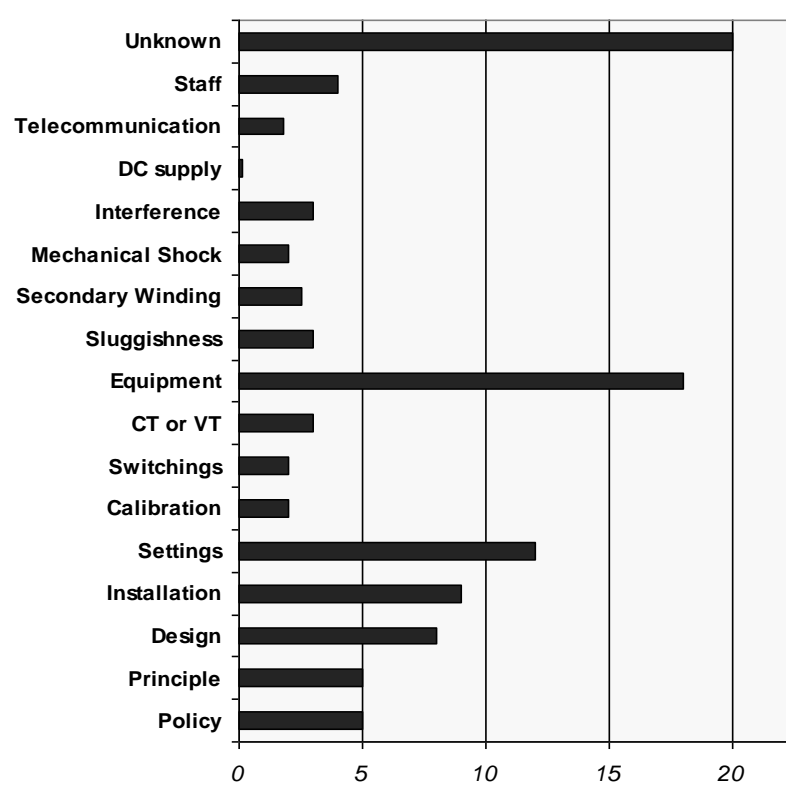

Fig. 1. Cause of Incorrect Protection Operation (1976-2002) [4]

\section{Power System Stability Simulation}

Practical realization of this concept is shown on Fig. 2. The EUROSTAG is used as power system regime simulator. Power system model represented with two generation areas

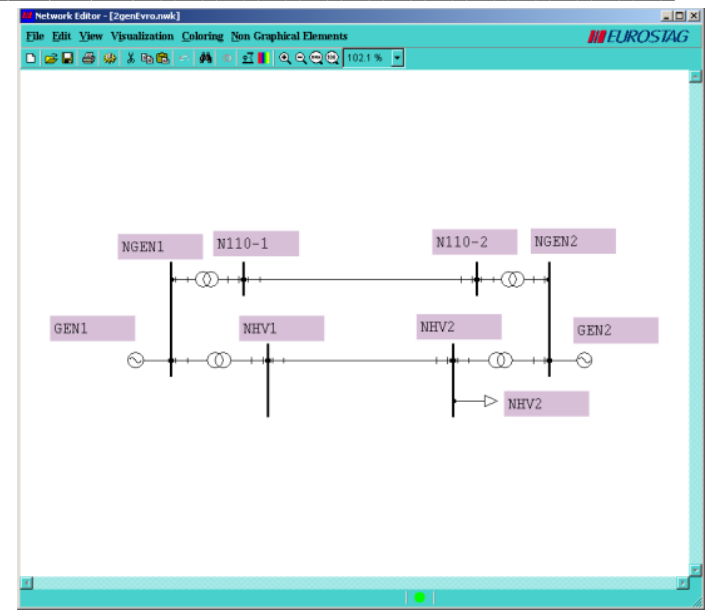

Fig. 2. Power system model used in stability study

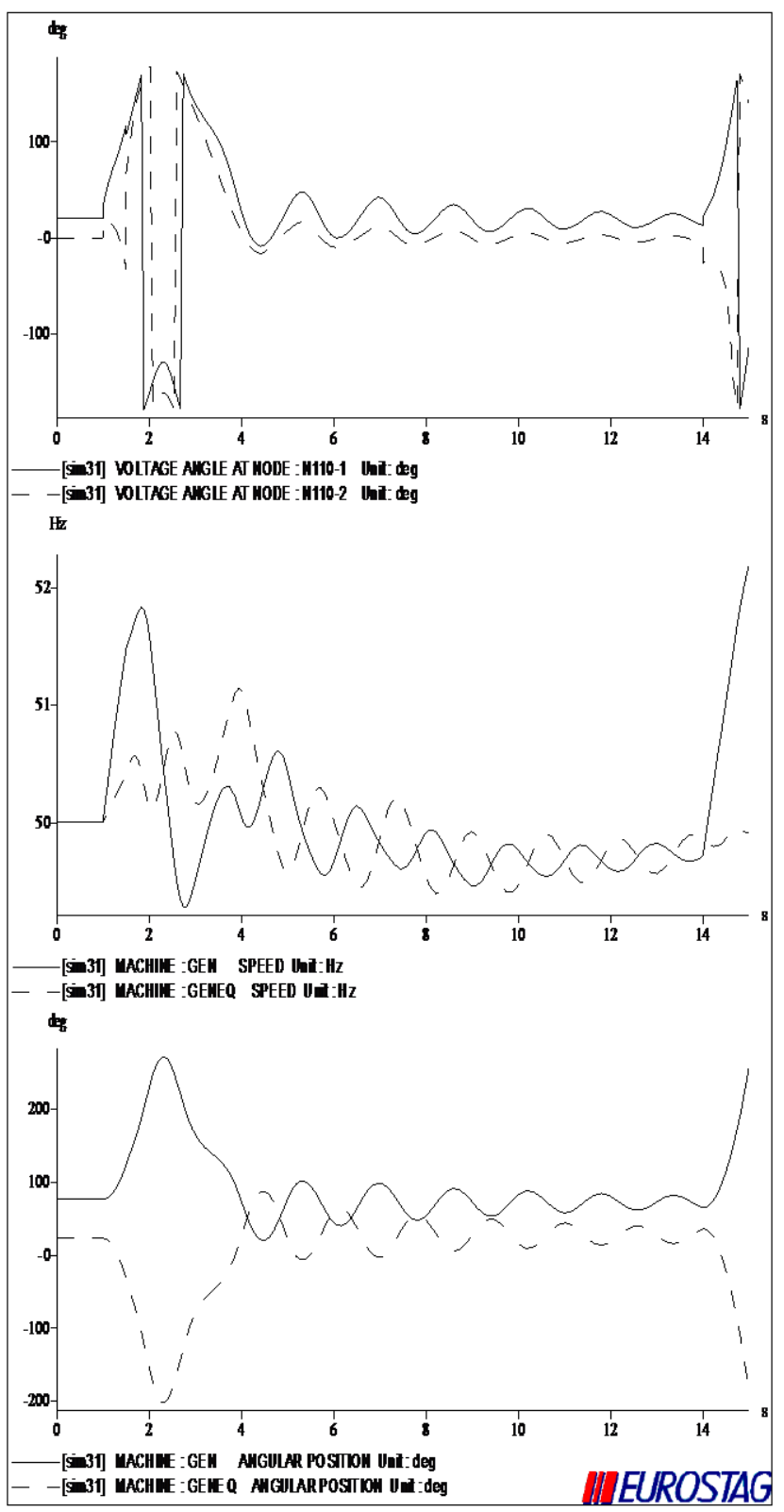

Fig. 3. Angular positions and voltage angles of GEN1, GEN2 generation area in the case of short circuit 
The power system can become unstable when short term loss of $330 \mathrm{kV}$ transmission link occurs. This condition can arise as a result of short circuit on $330 \mathrm{kV}$ line with a subsequent successful auto reclosing. Varying the short circuit clearing time, automatic reclosing delay, the load, short circuit type and location, different scenarios can be simulated. The behavior of the power angles for both generation areas of power system is presented on Fig. 3. Depending on the selected scenario, the cases from stable power swing toward the loss of synchronism and out-of-step condition can be simulated.

\section{OOS Relay “AGNA” Operation Principles}

The OOS relay should be used to protect the modelled power system from the out-of-step condition. For power swing condition detection and OOS relaying "AGNA" protection should be installed on N110-1 - N110-2 HV line. The operation conditions of the OOS protection AGNA are determined by one of the two algorithms. The first operation algorithm does not allow generator angle to slip and operation takes place in the first swing cycle. The second algorithm allows pole slip and device operates after some swing cycles depending on the settings. The power swing detection is based on the control of angle $\varphi$ between two simulated voltages $\underline{U}_{1}$ and $\underline{U}_{2}[6]$. To simulate these voltages two-machine circuit an equivalent of the real system - is used (Fig. 4).

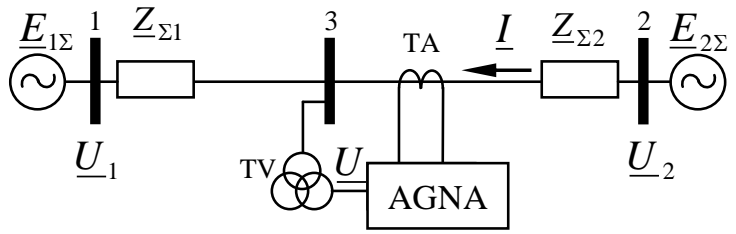

Fig. 4. Equivalent circuit of the power system

$$
\left.\begin{array}{l}
\frac{U_{1}}{U_{2}}=\underline{U} \pm \underline{Z}_{\Sigma 1} \cdot \underline{I} \\
\underline{U}-\underline{Z}_{\Sigma 2} \cdot \underline{I}
\end{array}\right\}
$$

where $\underline{U}$ and $\underline{I}$ are local voltage and current controlled by protection in the point of installation, $\underline{Z}_{\Sigma 1}$ and $\underline{Z}_{\sum 2}$ are the

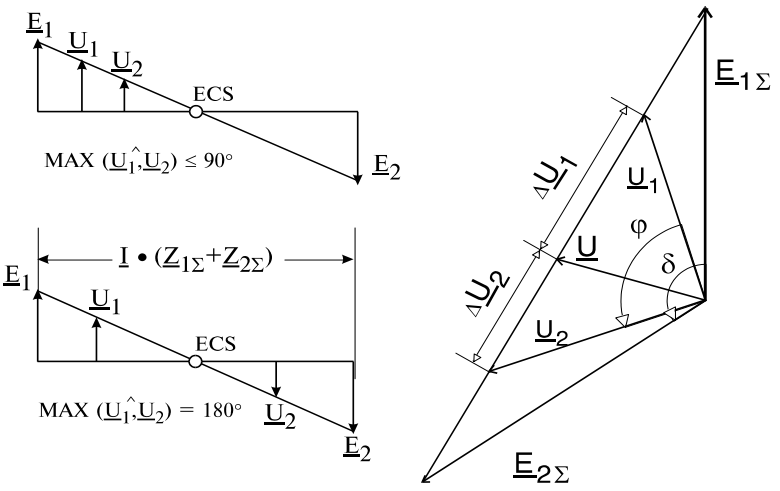

Fig. 5. Characteristic diagrams of device operation settings chosen depending on the equivalent parameters of the power system. Depending on the location of ESC (Fig. 5), the modelled voltages $\underline{U}_{1}$ and $\underline{U}_{2}$ can be located at the same side of ESC (the angle $\varphi$ does not exceed $90^{\circ}$ ) or on the opposite sides of ESC (angle $\varphi$ increases until it reaches $180^{\circ}$ ).

The protection operates when the following requirements are met:

1. angle $\varphi$ has reached its limit value;

2. angle changes with a sufficiently high rate $(\mathrm{d} \varphi / \mathrm{dt})$;

3. currents and voltages are symmetrical.

$$
\left.\begin{array}{l}
\varphi_{1}<\varphi=\arg \left(\frac{\underline{U}_{1}}{\underline{U}_{2}}\right)<\pi \\
C_{1}>d \varphi / d t>C_{2}
\end{array}\right\}
$$

where $\varphi_{1}, C_{1}$ and $C_{2}$ are the appropriate device settings. The device settings are preliminary calculated and can be checked and corrected during simulation if necessary.

Drawback of this voltage modelling principle is that voltage

$\dot{U}_{1}$ and $\dot{U}_{2}(1)$ is modelled using voltage and current in place of automation installing (bus 3), which can be situated far from generators. Measurement to be precise it is important to use EMF generators $\dot{E}_{1}$ and $\dot{E}_{2}$, which are situated after generators internal resistance. From bus 1 and 2 (fig. 4) different types of loads that raise auxiliary voltage drop on generator internal resistance can be supplied. That is why those bus voltages do not reproduce generator angles precisely.

Measurement structure of parameter measurement and EMF generator modelling (fig. 6) is free from the above mentioned drawback. EMF generator modelling process is described by formulas (3), (4) [7].

$$
\begin{aligned}
& \dot{E}_{1}=\dot{U}_{B 1}+\dot{I}_{G 1} \cdot \dot{Z}_{G 1}, \\
& \dot{E}_{2}=\dot{U}_{B 1} \dot{ }^{\bullet} \dot{I}_{L} \cdot \dot{Z}_{L}+\dot{I}_{G 2} \cdot \dot{Z}_{G 2},
\end{aligned}
$$

where $\dot{I}_{G 1}$ and $\dot{I}_{G 2}$ generator currents, $\dot{I}_{L}$ line current, $\dot{E}_{1}$ and $\dot{E}_{2}$ EMF generators, $\dot{Z}_{G 1}$ and $\dot{Z}_{G 2}$ resistances of branches generator-bus, $\dot{Z}_{L}$ line resistance, $\dot{U}_{K 1}$ bus voltage.

Current $\dot{I}_{G 2}$ measurements are transferred in relay instrument movement, which uses digital communication channel. Measurement synchronization in time is realized by GPS. Protection operation conditions are defined by formula (2). 


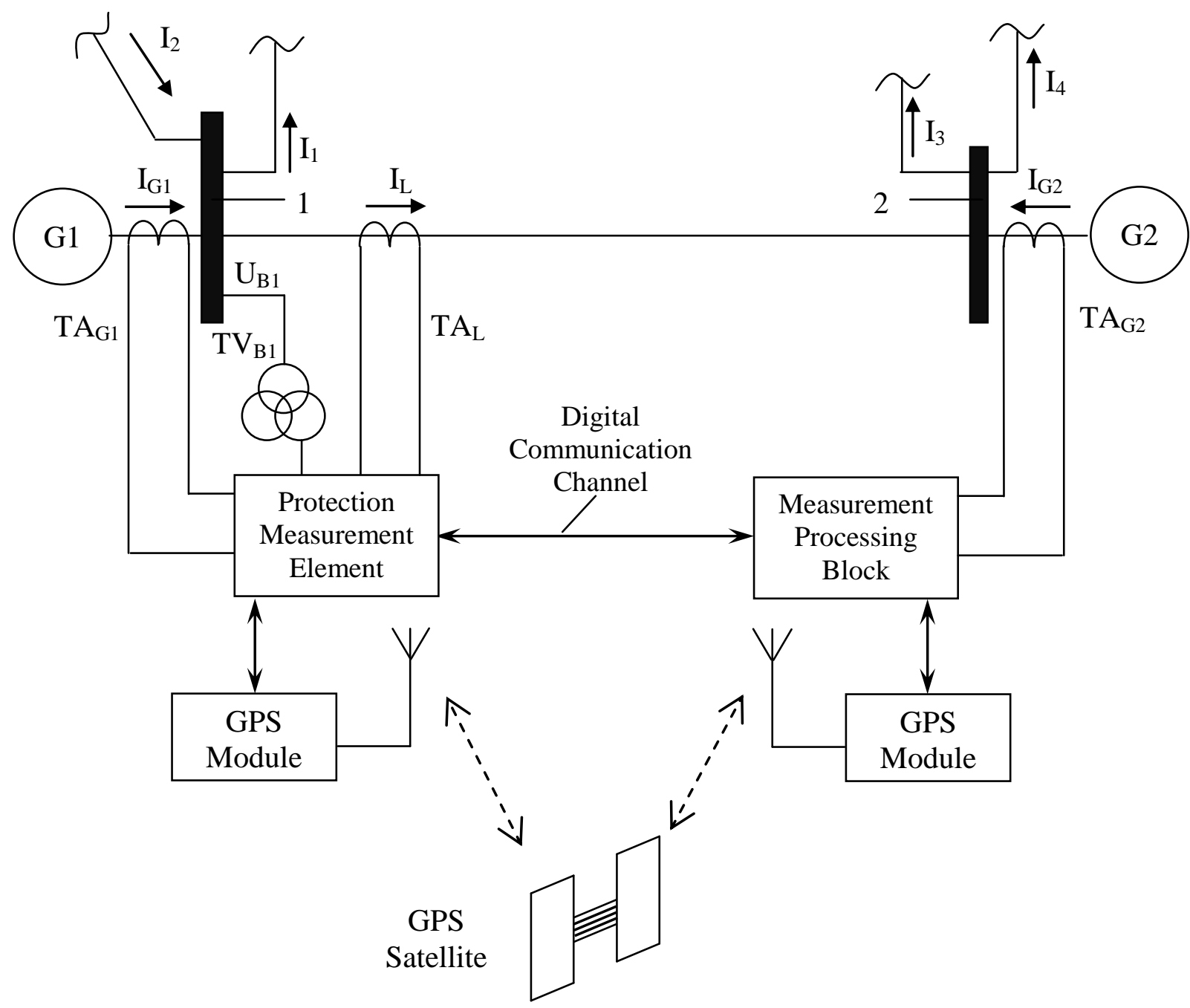

Fig. 6. Alternative method of OOS relay realization

\section{OOS Relay Testing with Power System Dynamics} Simulation Signals

The EUROSTAG allows any signal of power system dynamics simulation to be exported in ASCI format. Thus, 3phase currents and voltages, obtained during simulation, can be saved in external file and can be used (after conversion into real currents and voltages) for real device testing. The relay test system (ISA DRTS) is used for simulated signals playback.

Since the EUROSTAG output signals are represented with signal effective value and phase angle, but relay test system accept signals in COMTRADE format (instantaneous values), the converter program is needed.

Such conversion program was made and it converts the EUROSTAG output file into COMTRADE format.
As soon as the COMTRADE data file is obtained it can be used with any modern Relay Test Equipment which allows the signal waveforms playback. The complete picture is similar to one on Fig. 7. The power system model and various regimes (short circuits, line loss, load variations) are simulated with the EUROSTAG software. With the help of special program simulated signals are converted in COMTRADE format and uploaded in Relay Test System. The Relay Test System playback the currents and voltages and the reaction of the OOS relay AGNA is observed. Simulated out-of-step condition and AGNA OOS relay correct operation is presented on Fig. 8. AGNA output relay "2st trip" trip when the angle between simulated voltages $\mathrm{U} 1$ and $\mathrm{U} 2$ overreach the appropriate setting value. The waveforms analyses and device reaction for the particular experiment are made using the relay operation analyzing software "SMOKY". 


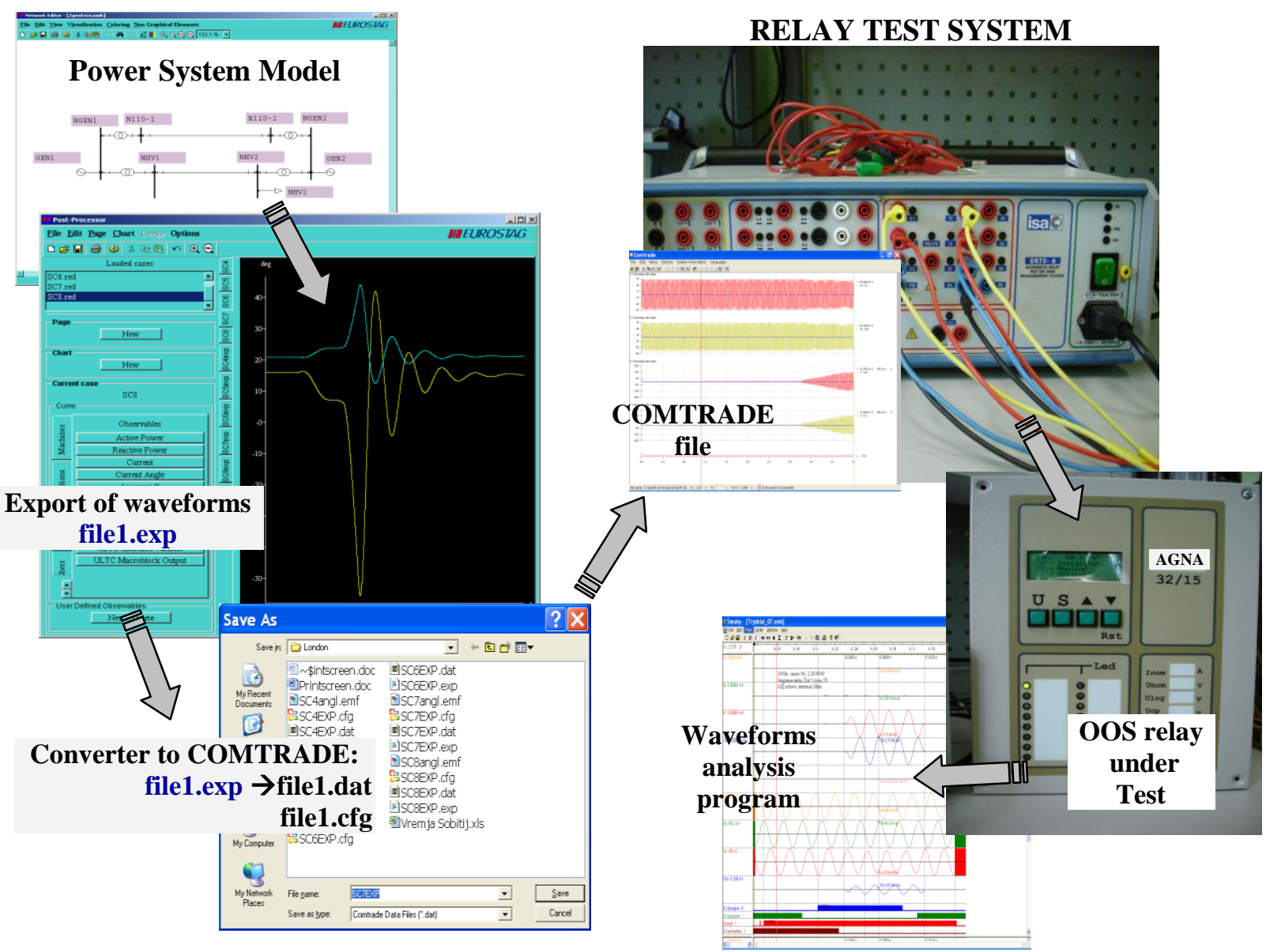

Fig. 7. OOS relay testing using simulation features of EUROSTAG

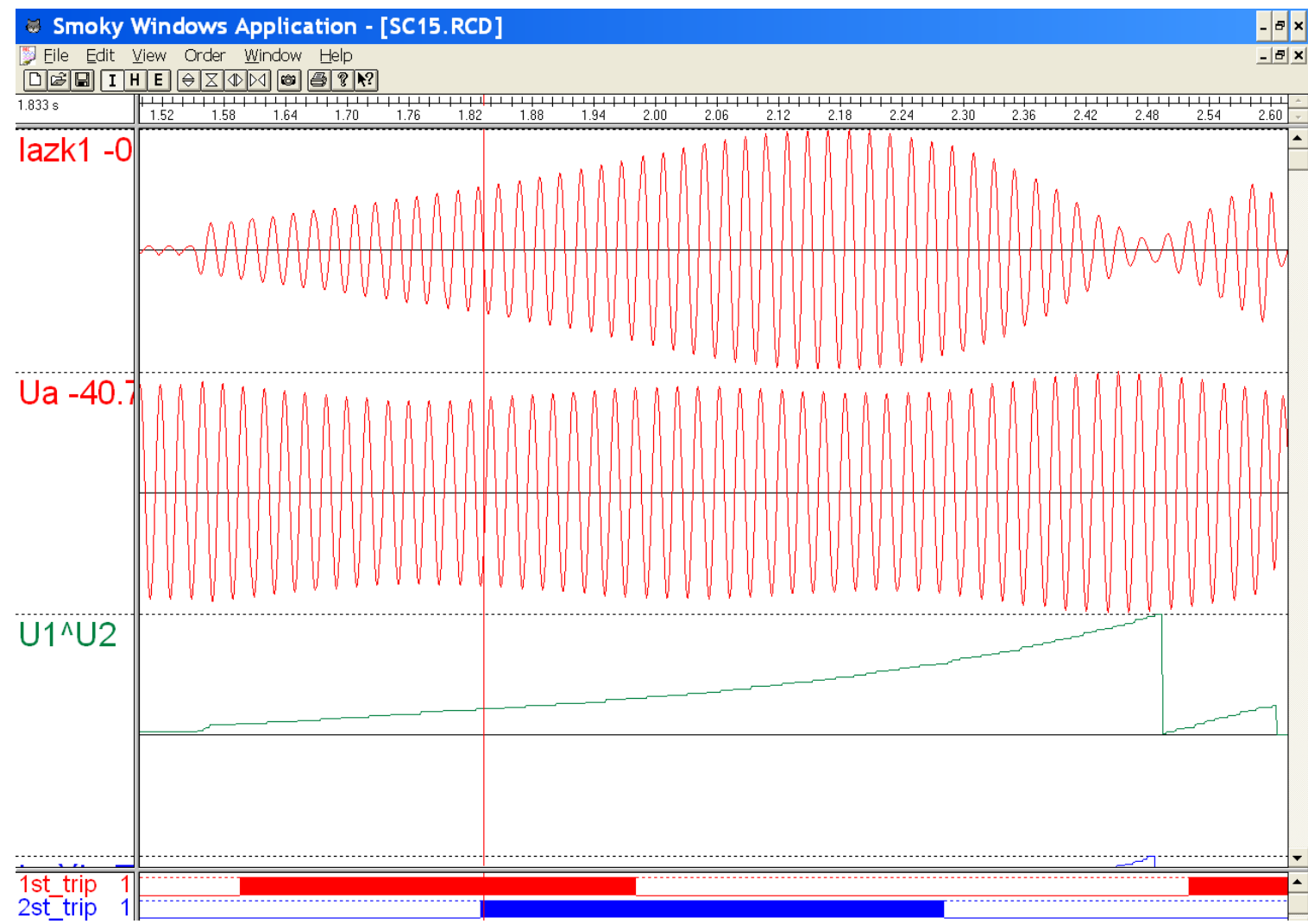

Fig. 8. Out-of-step regime simulation and OOS relay operation (2st_trip) 


\section{Extended Summary}

Large disturbances may cause loss of synchronism between some parts of power system and, if this condition ignored, can lead to widespread power outages and blackouts.

The extreme importance of OOS condition liquidation dictates that all possible efforts must be taken to guarantee the correct OOS relay operation.

The settings for OOS relay could be difficult to calculate because of the power system complexity and parameters variation in time. OOS relay operation should be verified in different power system regimes which hardly can be simulated using traditional relay testing technique. Power system dynamics modeling program EUROSTAG is used for power swing and out-of-step processes simulation.

Specially created program allows convert simulated signals into COMTRADE format and then real currents and voltages waveforms can be recreated by means of any modern relay test system.

Power system processes modeling technique was successfully implemented for protection and automation devices testing. Testing methodology allows the OOS relay to be tested in close-to-real conditions.

\section{References}

[1] E. W. Kimbark, Power System Stability, Vol 2, The Swing Equation and Its Solution, Wiley-IEEE Press, February 1995, p. 15-52.

[2] IEEE PSRC WG D6, Power Swing and Out-of-Step Considerations on Transmission Line, Final draft, June 2005, 59 p.

[3] M. A. Redfern, E. P. Walker, Power system simulation for the testing of protective equipment, Automatic Testing Conference, Brighton 1977 , Session 3, Vol 2, p. 143-158.

[4] T. Johannesson, F. Roos, S. Lindahl, Developments in Power System Protection, Eighth IEE International Conference, Vol 1, Sweden, 2004 p. 303-306.

[5] D. A. Tziouvaras, D. Hou, Out-of-Step Protection Fundamentals and Advancements, Proceedings of the 30th Annual Western Protective Relay Conference, Spokane, WA, October 21-23, 2003, 26 p.
[6] A. Sauhats, A. Svalov, Statistical Optimisation of a Complex of Local Devices for Prevention of Out-of-Step Conditions, 14th PSCC, Sevilla, 24-28 June 2002, Session 22, 5 p.

[7] A. Sauhats, D. Antonovs, A. Svalovs, J. Kucajevs, „OUT-OF-STEP RELAY”, Latvia's patent application Nr. P-11-67, May 12, 2011.

Antans Sauhats was born in Lithuania, on March 14, 1948. He received diploma engineer degree in 1970, Candidate of Technical Science degree (PhD) in 1976 and Dr. habil. sc. eng degree in 1991 from the Riga Technical University, Riga, Latvia (former Riga Polytechnic Institute). Since 1991 he is the Professor at Electric Power Systems. Since 1996 he is the Director of the Power Engineering Institute of the Riga Technical University.

His research interests include protective relaying and power system automation and control, power system emergency control, automation and relay protection as well as their mathematical modeling. He holds many patents in this area.

Andrejs Utans was born in Latvia, on April 6, 1964. He received diploma engineer degree in 1986, Candidate of Technical Science degree (PhD) in 1997 from Riga Technical University, Riga, Latvia. Since 2000 he is Docent at the Faculty of Electrical and Power Engineering. Since 2010 he is the associate Professor of the Power Engineering Institute of the Riga Technical University.

His research interests include protective relaying and power system automation and control.

Gregory Pashnin received Dipl.Eng. and Dr.sc.eng. degree from the Riga Technical University (former Riga Polytechnical Institute) in 1984 and 1992, respectively. Since 1991 he is researcher in the Power Engineering Institute of the Riga Technical University.

E-mail: pasnin@eef.rtu.lv

Dmitrijs Antonovs was born in Latvia, on May 6, 1986. He received B.Sc. and $M$. Sc. degree in electrical engineering from the Riga Technical University, Riga, Latvia, in 2008 and 2010, respectively.

$\mathrm{He}$ is a PhD. Student and reasercher at RTU, Power Engineering Institute, Riga, Latvia. His main scientific interests lay in power system risk assessment and management.

Address: Kronvalda blv., 1, LV-1010, Riga, Latvia

Phone: +37129741002

E-mail: d-lord@inbox.lv 